\title{
An Implementation Relation and Test Framework for Timed Distributed Systems ${ }^{\star}$
}

\author{
Christophe Gaston ${ }^{1}$, Robert M. Hierons ${ }^{2}$, and Pascale Le Gall ${ }^{3}$ \\ 1 CEA, LIST, Point Courrier 174, 91191, Gif-sur-Yvette, France \\ christophe.gaston@cea.fr \\ 2 Brunel University, Uxbridge, Middlesex, UK, UB8 3PH \\ rob.hierons@brunel.ac.uk \\ 3 Laboratoire MAS, Grande Voie des Vignes, 92195 Châtenay-Malabry, France \\ pascale.legall@ecp.fr
}

\begin{abstract}
Many systems interact with their environment at physically distributed interfaces and the distributed nature of any observations made is known to complicate testing. This paper concerns distributed testing, where a separate tester is placed at each localised interface and may only observe what happens at this interface. Most previous work on distributed model based testing has used models that are either finite state machines or input output transition systems. In this paper we define a framework for distributed testing from timed input output transition systems along with corresponding test hypotheses and a distributed conformance relation.
\end{abstract}

Keywords: distributed systems, timed systems, model based testing, symbolic input output transition systems.

\section{Introduction}

Most approaches to model based testing (MBT) assume that a single tester interacts with the system under test (SUT) and this tester observes all inputs and outputs. However, many systems such as web services and wireless sensor networks interact with the environment at multiple physically distributed interfaces. This has led to interest in distributed testing, where there is a separate local tester at each interface, a tester only observes events at its interface, and there is no global clock. This approach to distributed testing was formalised by ISO as the distributed test architecture [1. It is known that the use of the distributed test architecture affects software testing [2 7]. However, only recently has the effect been formalised as implementation relations for FSMs [8] and IOTS:1] [10, 11].

* This work was partially supported by the French Program "Investissements d'Avenir" in the IRT/SystemX/FSF project and the SesamGrid project, and by the ITEA2 project openETCS.

1 The implementation relation mioco [9] has also been defined for testing systems with distributed interfaces but this assumes that a single tester makes global observations. 
Previous work showed that the distributed test architecture causes additional controllability and observability problems. A controllability problem is a situation where a local tester does not know when to apply an input [2. Consider, for example, a test case where tester $t_{1}$ at interface 1 applies input $i_{1}$ ?, this should lead to output $o_{1}$ ! at 1 , and tester $t_{2}$ at interface 2 should then send input $i_{2}$ ?. Here $t_{2}$ cannot know when to send $i_{2}$ ? since it does not observe the previous input and output. Observability problems refer to situations where one cannot distinguish between the global trace produced by the SUT and that expected despite these being different [3]. Let us suppose, for example, that the specification contains global trace $\sigma$ where the response to a first $i_{1}$ ? at interface 1 leads to output $o_{1}$ ! at 1 , and a second $i_{1}$ ? leads to output $o_{1}$ ! at 1 and output $o_{2}$ ! at 2 . Here, the tester at 1 expects to observe $i_{1} ? o_{1} ! i_{1} ? o_{1}$ ! and the tester at 2 expects to observe $\mathrm{O}_{2}$ ! and this observation is made if the SUT produces global trace $\sigma^{\prime}$ in which $o_{2}$ ! is output in response to the first input rather than the second. While $\sigma$ and $\sigma^{\prime}$ are different they have the same projections at the interfaces and so it is not possible to distinguish between them in distributed testing.

This paper explores distributed testing from systems described by means of cooperating timed input output transition systems (TIOTS): IOTS extended with time. We assume that testers have local clocks that are not synchronised but clocks progress at the same rate; it should be straightforward to adapt this to the case where the clocks can drift. As far as we are aware only two previous papers have explored the role of clocks/time in distributed testing and these consider different problems. One paper uses timestamps and bounds on clock differences to strengthen implementation relations for IOTSs 12 but did not consider timed models. A second looked at coordinating distributed testing from an FSM through the testers exchanging messages when we have bounds on message latency 13 . While this considered timed models it assumed that the model is an FSM with time and concentrated on overcoming controllability problems.

The previously defined implementation relation dioco for distributed testing against an IOTS compares global traces of the SUT against global traces of the specification using $\sim$ where $\sigma \sim \sigma^{\prime}$ denotes $\sigma$ and $\sigma^{\prime}$ having the same local projections. In order to ensure that the observations at the interfaces are all projections of the same global trace, it considers either global traces of the SUT that end in quiescence 2 or infinite traces of the SUT [10,12.

This paper uses an alternative approach in which an observation is a tuple $\left(\sigma_{1}, \ldots, \sigma_{n}\right)$ where the tester at $p$ observes $\sigma_{p}$ and we call such an observation a multitrace. Instead of defining the implementation relation with some projection mechanisms, we directly use the notion of multitraces to define a new implementation relation dtioco for distributed testing. We also provide a compositionality result, which says that dtioco holds if and only if all multitraces of the SUT are such that exchanged messages respect some communication rules and also that the local projections of the SUT conform to the local components of the

${ }^{2}$ The SUT is quiescent if it cannot produce output without receiving input. 
specification under tioco. This allows standard techniques for tioco [14,15] to be used in distributed testing.

Having defined a new implementation relation we describe a test architecture for TIOTSs such that test cases can be denoted as multitraces. In distributed testing we have to bring together observations made by the local testers in order to determine whether the SUT has passed a test case. Solving the oracle problem mainly becomes a multitrace analysis problem and is described as a two step process, in which a test case is run and then a verdict is produced based on the set of local (timed) traces observed. We then describe how timed testing can be carried out and provide an algorithm for checking that communication rules are verified; the compositionality result tells us that we can derive a verdict using such an algorithm along with standard methods for tioco.

The paper is structured as follows. Section 2 defines the terminology and notation used in this paper and in Section 3 we describe TIOTSs. In Section 4, we present specifications of timed distributed systems as a collection of cooperating TIOTSs. Section 5 gives the test framework, defines the new implementation relation dtioco and provides an example. Section 6 then explains how distributed timed testing can be carried out. Finally, Section 7 draws conclusions and discusses possible avenues for future work.

\section{Preliminaries}

We will use a carrier set $D$ (for Duration) isomorphic to the set of strictly positive real numbers 3 . We may use classical operations,$+-: D \times D \rightarrow D,<, \leq$ : $D \times D \rightarrow$ bool... on durations 4 , provided by default with their usual meanings. Variables having their values in $D$ are called clocks.

A Labelled Transition Systems (LTS) $\mathbb{G}$ over a set of labels $L$ is a triple $\left(Q, q_{i n}, T\right)$ where $Q$ is a set of states, $q_{i n} \in Q$ is the initial state, and $\operatorname{Tr} \subseteq$ $Q \times L \times Q$ is a set of transitions. For transition $\operatorname{tr}=\left(q, a, q^{\prime}\right)$, also denoted $q \stackrel{a}{\rightarrow} q^{\prime}$, source $(t r)$ stands for $q$, target $(t r)$ stands for $q^{\prime}$ and $\operatorname{act}(t)$ stands for the action a. Paths $(\mathbb{G}) \subseteq T^{*}$ is 5 the set of paths of $\mathbb{G}$ which contains the empty sequence $\varepsilon$ and all sequences of transitions $t r_{1} \cdots t r_{n}$ with $n \geq 1$ such that $\operatorname{source}\left(\operatorname{tr}_{1}\right)=q_{\text {in }}$ and for all $i$ satisfying $1<i \leq n$, we have $\operatorname{source}\left(\operatorname{tr}_{i}\right)=\operatorname{target}\left(\operatorname{tr}_{i-1}\right)$. For any $p$ in path Paths $(\mathbb{G})$, the trace of $p$, denoted trace $(p)$, is inductively defined as $\varepsilon$ for $p=\varepsilon$, and act $(t r)$.trace $\left(p^{\prime}\right)$ for $p=t r . p^{\prime}$ with $t r$ a transition and $p^{\prime}$ a path. $\operatorname{Traces}(\mathbb{G})$ stands for the set of traces of all paths of Paths $(\mathbb{G})$.

\section{Timed Input Output Transition Systems}

In this section we define Timed Input Output Transition Systems (TIOTSs) and associated notation. We then discuss and formalise as multitraces the

\footnotetext{
${ }^{3}$ In practice, any set of values used in a constraint solver for approaching real numbers.

${ }^{4} d_{1}-d_{2}$ is defined if and only if $d_{1}>d_{2}$.

${ }^{5}$ Given a set $A, A^{*}$ denotes the set of finite sequences of elements of $A$, $\varepsilon$ denotes the empty sequence, and '? is used for concatenation.
} 
observations that may be made in distributed testing. TIOTSs are labelled transition systems whose labels represent either Outputs, Inputs or durations.

Definition 1 (TIOTS). A TIOTS-signature is a tuple $\Sigma=(C, I, O)$ with $I \cap$ $O=\emptyset$, where $C$ is a set of channels, $I$ is a set of inputs and $O$ is a set of outputs. Moreover $C$ can be partitioned as $C_{\text {in }} \coprod C_{\text {out }}$ where $C_{\text {in }}$ and $C_{\text {out }}$ are a set of input channels and a set of output channels respectively. Accordingly, $I$ and $O$ can be partitioned as $\coprod_{c \in C_{\text {in }}} I_{c}$ and $\coprod_{c \in C_{\text {out }}} O_{c}$ respectively, where for channel $c$ we have that $I_{c}$ is the set of inputs that can be received on $c$ and $O_{c}$ is the set of outputs that can be sent through $c$. A TIOTS over $\Sigma$ is an LTS $\left(Q, q_{\text {in }}, T\right)$ over $I \cup O \cup D$.

In the sequel, for any TIOTS $\mathbb{A}$ over $\Sigma=(C, I, O), \operatorname{Sig}(\mathbb{A})$ stands for $\Sigma$, $C(\mathbb{A})$ or $C(\Sigma)$ stand for $C, I(\mathbb{A})$ or $I(\Sigma)$ stand for $I$, and $O(\mathbb{A})$ or $O(\Sigma)$ stands for $O$. Moreover, in a slight abuse of notation we use $\Sigma^{*}$ for $(I \cup O \cup D)^{*}$. Inputs (respectively Outputs) occuring in $I_{c}$ (respectively $O_{c}$ ) are sometimes denoted $c ? a$ (respectively $c ! a$ ) where $a$ is a value received (sent) through channel $c$. We also use $c$ ? or $c$ ! for simple signals received or sent through channel $c$. Executions of TIOTS are called Timed Traces, which are defined as follows:

Definition 2 (Timed Traces). The set TTraces $(\mathbb{A})$ of timed traces of the TIOTS $\mathbb{A}$ is the smallest set that satisfies the following:

- for any $\sigma \in \operatorname{Traces}(\mathbb{A})$ (with $\mathbb{A}$ viewed as a simple LTS), if $\sigma$ is of the form $\nabla . \sigma^{\prime}$ where $\nabla=d_{1} \ldots d_{n}, n \geq 0$, for all $i \leq n, d_{i} \in D$, and $\sigma^{\prime}$ is either $\varepsilon$ or of the form a. $\sigma^{\prime \prime}$ with $a \in I \cup O$, then $\sigma^{\prime} \in \operatorname{TTraces}(\mathbb{A})$.

- for any $d_{1}, d_{2}$ and $d_{3}$ in $D$ satisfying $d_{1}+d_{2}=d_{3}$, for any $\sigma, \sigma^{\prime}$ in $\Sigma^{*}$, $\sigma . d_{3} . \sigma^{\prime} \in \operatorname{TTraces}(\mathbb{A})$ iff $\sigma \cdot d_{1} \cdot d_{2} . \sigma^{\prime} \in \operatorname{TTraces}(\mathbb{A})$.

Given timed trace $\sigma$ and action $a$ in $I \cup O,|\sigma|_{a}$ will denote the number of instances of $a$ in $\sigma$.

The first point of the definition prevents us from having timed traces beginning with durations. This is because in black box testing we cannot differentiate between the SUT not being initialised from the SUT being initialised but not having interacted with its environment. Thus, if $d$ is a duration then we cannot distinguish between traces $d . \sigma$ and $\sigma$. In a distributed context, this problem is even more pronounced since we cannot even expect that the tester and SUT are initially synchronised via a reset. The second point says that durations can be composed and decomposed provided that cumulative sums of consecutive durations are maintained. The definition of timed traces makes no assumption on how durations are sampled in testing (it allows all possible choices).

\section{Specifications of Timed Distributed Systems}

We specify systems as a collection of localized parts (described as TIOTSs) communicating through a network. In particular, each localisation $l$ is identified by its

${ }^{6}$ If $n=0$, then $\nabla=\varepsilon$. 
interface, given as a TIOTS signature $\Sigma_{l}$. The next definition characterises communications between a collection of interfaces, that is a system signature. They are given as a set of consistency conditions defining tuples of local executions (timed traces) corresponding to compatible visions of some global execution:

Definition 3 (System communications). A system signature $\Sigma_{S y s}$ is a tuple $\left(\Sigma_{1}, \ldots, \Sigma_{n}\right)$ of TIOTS signatures. The set of multitraces over $\Sigma_{\text {Sys }}$, denoted $\operatorname{MTraces}\left(\Sigma_{\text {Sys }}\right)$, is the subset of $\Sigma_{1}^{*} \times \ldots \times \Sigma_{n}^{*}$ defined as follows:

Empty Trace: $(\varepsilon \ldots \varepsilon)$ is in $\operatorname{MTraces}\left(\Sigma_{S y s}\right)$,

Inputs from the environment: for any $i \leq n, c \in C\left(\Sigma_{i}\right) \backslash \cup_{j \neq i} C\left(\Sigma_{j}\right), a \in$ $I\left(\Sigma_{i}\right)_{c}$, and $\left(\sigma_{1} \ldots \sigma_{i}, \ldots \sigma_{n}\right) \in \operatorname{MTraces}\left(\Sigma_{S y s}\right)$ we have $\left(\sigma_{1} \ldots \sigma_{i} . a, \ldots \sigma_{n}\right) \in$ $\operatorname{MTraces}\left(\Sigma_{\text {Sys }}\right)$.

Non Blocking Outputs: for any $i \leq n, a \in O\left(\Sigma_{i}\right)$, and $\left(\sigma_{1} \ldots \sigma_{i}, \ldots \sigma_{n}\right) \in$ $\operatorname{MTraces}\left(\Sigma_{\text {Sys }}\right)$, we have $\left(\sigma_{1} \ldots, \sigma_{i} . a, \ldots \sigma_{n}\right) \in \operatorname{MTraces}\left(\Sigma_{\text {Sys }}\right)$.

Causality of communication: for any $i \leq n, a \in I\left(\Sigma_{i}\right)_{c}$ where $c$ is a channel of at least two TIOTS-signatures of Sys, and $\left(\sigma_{1} \ldots, \sigma_{i}, \ldots \sigma_{n}\right) \in$ $\operatorname{MTraces}\left(\Sigma_{\text {Sys }}\right)$, let us denote by $O_{a} \subseteq\{1, \ldots, n\}$ the set of all indexes $j$ such that $a \in O\left(\Sigma_{j}\right)_{c}$. If $\left|\sigma_{i}\right|_{a}<\Sigma_{j \in O_{a}}\left|\sigma_{j}\right|_{a}$, then $\left(\sigma_{1} \ldots, \sigma_{i} . a, \ldots, \sigma_{n}\right) \in$ $\operatorname{MTraces}\left(\Sigma_{\text {Sys }}\right)$.

Consistent Time Elapsing: for any $d \in D$ and $\left(\sigma_{1}, \ldots, \sigma_{n}\right) \in \operatorname{MTraces}\left(\Sigma_{S y s}\right)$, we have $\left(\sigma_{1}^{\prime}, \ldots, \sigma_{n}^{\prime}\right) \in \operatorname{MTraces}\left(\Sigma_{\text {Sys }}\right)$ where for any $i \leq n, \sigma_{i}^{\prime}$ is equal to $\varepsilon$ if $\sigma_{i}=\varepsilon$ and equal to $\sigma_{i} . d$ otherwise.

A multitrace is a tuple, each element being a sequence of inputs, outputs or durations that is an execution that may be observed on a localised interface. The multitrace whose sequences are all $\varepsilon$ (Item Empty Trace) corresponds to no interaction having occurred. A multitrace can be extended by adding to any component either an input from the environment (Item Inputs from the environment) or an output (Item Non Blocking Outputs). Outputs are non-blocking, when sent to the environment and when sent to other parts of the system. Internal communications are on shared channels. A channel may be shared by an arbitrary number of localised TIOTSs. Internal communication is multicast: a message sent can be received by several recipients (all those who listen on the channel of interest). Messages are never lost but the time to reach a recipient is not quantifiable since it travels between interfaces and there is no global clock (we cannot measure it). If we focus on a thread of execution (i.e. a sequence in a multitrace), a message cannot be received more often than the total number of emissions of this message in the system (Item Causality of communication). Finally, we require that time elapses in the same way for all interfaces whose corresponding trace is not empty (Item Consistent Time Elapsing).

In distributed testing there is a separate localised tester at each interface and there is no global clock. Thus, we cannot make any suppositions on the different moments at which the different testers stop observing their associated interfaces. To reflect this, we accept as admissible observations multitraces made of trace prefixes, which we call observable multitraces. 
Definition 4 (Observable Multitraces). The set of observable multitraces of $\Sigma_{\text {Sys }}=\left(\Sigma_{1} \ldots \Sigma_{n}\right)$, denoted OTraces $\left(\Sigma_{\text {Sys }}\right)$, is the smallest set containing MTraces $\left(\Sigma_{\text {Sys }}\right)$ and such that for any a in $\Sigma_{i}$, we have:

$\left(\sigma_{1}, \ldots, \sigma_{i} . a, \ldots, \sigma_{n}\right) \in \operatorname{OTraces}\left(\Sigma_{\text {Sys }}\right) \Rightarrow\left(\sigma_{1}, \ldots, \sigma_{i}, \ldots, \sigma_{n}\right) \in \operatorname{OTraces}\left(\Sigma_{\text {Sys }}\right)$

On each localised subsystem, the observer only observes a prefix of the whole (local) timed trace if it does not wait long enough. Now, system specifications are defined as tuples $\left(\mathbb{A}_{1}, \ldots, \mathbb{A}_{n}\right)$ of TIOTSs whose associated observable multitraces $\left(\sigma_{1} \ldots, \sigma_{i}, \ldots \sigma_{n}\right)$ are those such that $\sigma_{i}$ is a timed trace of $\mathbb{A}_{i}$.

Definition 5 (System). A system Sys over $\Sigma_{S y s}=\left(\Sigma_{1}, \ldots, \Sigma_{n}\right)$ is a tuple $\left(\mathbb{A}_{1}, \ldots, \mathbb{A}_{n}\right)$ of TIOTS, $\mathbb{A}_{i}$ being defined on $\Sigma_{i}(1 \leq i \leq n)$. OTraces $($ Sys $)$ is the set of multitraces:

$$
\left(\operatorname{TTraces}\left(\mathbb{A}_{1}\right) \times \ldots \times \operatorname{TTraces}\left(\mathbb{A}_{n}\right)\right) \cap \operatorname{OTraces}\left(\Sigma_{\text {Sys }}\right)
$$

Each TIOTS corresponds to a view of the system from one interface. Its timed traces denote possible observations of system executions from this interface. Observable traces denote tuples of consistent views of system executions.

\section{Testing Framework}

\subsection{A Conformance Relation for Timed Distributed Systems}

In this section we define our new implementation relation dtioco. In MBT it is normal to assume that certain test hypotheses hold [16, the most basic hypothesis being that the SUT can be described using the same formalism as the specification. We assume that the following classical test hypotheses hold.

Definition 6 (LUT and SUT). Let $\Sigma=(C, I, O)$ be a TIOTS signature and $C^{\prime} \subseteq C$. A TIOTS $\mathbb{A}=\left(Q, q_{\text {in }}, T r\right)$ over $\Sigma$ satisfies the so-called Input Enableness property over $C^{\prime}$ iff $\forall q \in Q, \forall c \in C^{\prime}, \forall a \in I_{c}, \exists q^{\prime} \in Q,\left(q, a, q^{\prime}\right) \in T r$.

$A$ Localized System Under Test (LUT) over $\left(\Sigma, C^{\prime}\right)$ is a TIOTS over $\Sigma$, satisfying the input enableness property over $C^{\prime}$.

$A$ System Under Test (SUT) over $\Sigma_{\text {Sys }}=\left(\Sigma_{1}, \ldots, \Sigma_{n}\right)$ is a tuple $\left(\mathbb{L S}_{1}, \ldots, \mathbb{L S}_{n}\right)$ such that for each $1 \leq i \leq n, \mathbb{L S}_{i}$ is an LUT over $\left(\Sigma_{i}, I\left(\Sigma_{i}\right) \backslash\left(\cup_{j \in 1 . . n} O\left(\Sigma_{j}\right)\right)\right)$.

Input-enabledness adapts the traditional hypothesis to distributed testing by requiring that the system is input-enabled on its public interface made of channels shared with the environment. We base our new conformance relation on tioco [14, 17 19. In fact, we use a slightly modified version of tioco since, as stated in Definition 2, our timed traces start with an input or an output (we remove durations occuring at the beginning of traces).

Definition 7 (tioco). Let $\mathbb{L S}$ be an LUT and $\mathbb{A}$ a TIOTS both defined on the same signature $(C, I, O)$. $\mathbb{L}$ S conforms to $\mathbb{A}$, denoted $\mathbb{L} \mathbb{S}$ tioco $\mathbb{A}$, if and only if for any $\sigma$ in TTraces $(\mathbb{A})$ and $r$ in $O \cup D$, we have:

$$
\sigma . r \in \operatorname{TTraces}(\mathbb{L} \mathbb{S}) \Longrightarrow \sigma . r \in \operatorname{TTraces}(\mathbb{A})
$$


Our conformance relation for distributed systems is an extension of tioco to observable multitraces, except that observable multitraces introduce some constraints (Definition 3), typically on internal receptions that should be preceded by internal emissions. However, it may happen that an observation of an SUT $\mathbb{S}$ does not satisfy those constraints. For example, the network might create a spurious message in a channel between localised systems, a localised system $\mathbb{L S}_{i}$ receiving a message (input) on a channel $c$ that connects it to another localised system $\mathbb{L S}_{j}$ without $\mathbb{L}_{\mathbb{S}_{j}}$ sending this message. Let us note that there exists no specification to which such systems conform according to Definition 8 since they do not meet consistency conditions of Definition 3 . Although our implementation relation will consider such behaviours to be erroneous, we cannot assume that the possible executions of the SUT are in OTraces $\left(\Sigma_{S y s}\right)$. In fact, we will only suppose that each local execution is a timed trace of some localised system $\mathbb{L S}_{i}$ under test. In the sequel any SUT $\mathbb{S}=\left(\mathbb{L}_{1}, \ldots, \mathbb{L}_{n}\right)$ has a set $\operatorname{Sem}(\mathbb{S}) \subseteq \operatorname{TTraces}\left(\mathbb{L}_{1}\right) \times \ldots \times \operatorname{TT}$ Taces $\left(\mathbb{L}_{n}\right)$ that denotes the set of all observations that can be made in testing; this allows the network to introduce messages on channels as discussed earlier. We further suppose, as in the case of observable traces, that for any $a$ we have that: $\left(\left(\sigma_{1}, \ldots, \sigma_{i} . a, \ldots, \sigma_{n}\right) \in \operatorname{Sem}(\mathbb{S})\right) \Rightarrow\left(\left(\sigma_{1}, \ldots, \sigma_{i}, \ldots, \sigma_{n}\right) \in \operatorname{Sem}(\mathbb{S})\right)$.

Definition 8 (dtioco). Let $\mathbb{S}=\left(\mathbb{L}_{1}, \ldots, \mathbb{L}_{n}\right)$ and $S y s=\left(\mathbb{A}_{1}, \ldots, \mathbb{A}_{n}\right)$ be resp. an SUT and a system both on the signature $\Sigma_{S y s}=\left(\Sigma_{1}, \ldots, \Sigma_{n}\right)$.

$\mathbb{S}$ conforms to Sys, denoted $\mathbb{S}$ dtioco Sys, if and only if $\operatorname{Sem}(\mathbb{S}) \subseteq \operatorname{OTrace}\left(\Sigma_{\text {Sys }}\right)$ and for any $\left(\sigma_{1} \ldots, \sigma_{i}, \ldots, \sigma_{n}\right) \in \operatorname{OTraces}(\operatorname{Sys})$ and $r \in\left(\cup_{i \leq n} O\left(\Sigma_{i}\right)\right) \cup D$, we have:

$$
\left(\sigma_{1} \ldots, \sigma_{i} . r, \ldots \sigma_{n}\right) \in \operatorname{Sem}(\mathbb{S}) \Longrightarrow\left(\sigma_{1} \ldots, \sigma_{i} . r, \ldots \sigma_{n}\right) \in \operatorname{OTraces}(\operatorname{Sys})
$$

The first part of the definition requires that any observation of the SUT is valid. The second part follows an approach similar to tioco in that it says that for any observable multitrace $\left(\sigma_{1} \ldots \sigma_{i}, \ldots, \sigma_{n}\right)$ of the specification, every possible next observation of the SUT after $\left(\sigma_{1} \ldots \sigma_{i}, \ldots, \sigma_{n}\right)$ is also an observation that might be made after $\left(\sigma_{1} \ldots \sigma_{i}, \ldots, \sigma_{n}\right)$ in the specification.

In the sequel, we introduce a compositionality result that allows us to reuse testing algorithms dedicated for tioco in a distributed system testing process. We begin by introducing a definition allowing us to identify which part of an LUT is stimulated in a distributed SUT.

Definition 9. Let $\mathbb{S}=\left(\mathbb{L}_{\mathbb{S}_{1}}, \ldots, \mathbb{L}_{\mathbb{S}_{n}}\right)$ be an $S U T$ with $\mathbb{L}_{i}=\left(Q_{i}, q_{i}, T r_{i}\right)$ for $i$ in 1 ..n. The projection of $\mathbb{L S}_{i}$ on $\mathbb{S}$, denoted $\left.\mathbb{L S}_{i}\right|_{\mathbb{S}}$ is the TIOTS $\left(Q_{i}, q_{i}, \operatorname{Tr}_{i}^{\prime}\right)$ where $\operatorname{Tr}_{i}^{\prime}$ is the subset of $\operatorname{Tr}_{i}$ that contains all transitions $t r$ such that there exists a path of the form $p^{\prime} . t$ in Paths $\left(\mathbb{L S}_{i}\right)$, a timed trace $\sigma$ in TTraces $\left(p^{\prime} . t r\right)$ and a tuple $\left(\sigma_{1}, \ldots, \sigma_{n}\right)$ in $\operatorname{Sem}(\mathbb{S})$ such that $\sigma=\sigma_{i}$.

The set of timed traces of $\left.\mathbb{L} \mathbb{S}_{i}\right|_{\mathbb{S}}$ contains all timed traces of $\mathbb{L} \mathbb{S}_{i}$ that $\mathbb{L} \mathbb{S}_{i}$ can produce when interacting with the other LUTs. Thus, if a tester interacts with $\mathbb{S}$ only through the channels of $\mathbb{L} \mathbb{S}_{i}$, he/she interacts with a real system that may be represented by $\left.\mathbb{L}_{\mathbb{S}_{i}}\right|_{\mathbb{S}}$ (except that the tester does not control inputs received 
on channels between LUTs). By construction, $\left.\mathbb{L S}_{i}\right|_{\mathbb{S}}$ need not be input enabled over all internal channels since all configurations over the localised part are not exercised in the context of $\mathbb{S}$.

Property 1. Let $\mathbb{S}=\left(\mathbb{L}_{1}, \ldots, \mathbb{L} \mathbb{S}_{n}\right)$ and Sys $=\left(\mathbb{A}_{1}, \ldots, \mathbb{A}_{n}\right)$ be resp. an SUT and a system with both having signature $\Sigma_{S y s}=\left(\Sigma_{1}, \ldots, \Sigma_{n}\right)$.

If for all $i$ in $1 . . n, \mathbb{A}_{i}$ is input enabled over $C\left(\Sigma_{i}\right)$, the following result holds: $(\mathbb{S}$ dtioco Sys $) \Leftrightarrow\left(\left(\forall i \leq n,\left.\mathbb{L}_{i}\right|_{\mathbb{S}}\right.\right.$ tioco $\left.\left.\mathbb{A}_{i}\right) \wedge \operatorname{Sem}(\mathbb{S}) \subseteq \operatorname{OTraces}\left(\Sigma_{\text {Sys }}\right)\right)$

Proof. First consider the left-to-right implication. The fact that $\operatorname{Sem}(\mathbb{S}) \subseteq$ OTraces $\left(\Sigma_{S y s}\right)$ is part of the definition of dtioco.

Now let us suppose that there exists $i$ in $1, \ldots, n$ such that $\neg\left(\left.\mathbb{L}_{i}\right|_{\mathbb{S}}\right.$ tioco $\left.\mathbb{A}_{i}\right)$. If so, there exists $\sigma_{i} \in \operatorname{Traces}\left(\mathbb{A}_{i}\right)$ and $r \in O\left(\Sigma_{i}\right) \cup D$ such that $\sigma_{i} . r \in$ $\operatorname{TTraces}\left(\left.\mathbb{L}_{\mathbb{S}_{i}}\right|_{\mathbb{S}}\right)$ and $\sigma_{i} . r \notin \operatorname{TTraces}\left(\mathbb{A}_{i}\right)$.

Since $\sigma_{i} . r \in \operatorname{Traces}\left(\left.\mathbb{L}_{\mathbb{S}_{i}}\right|_{\mathbb{S}}\right)$, there exists $\left(\sigma_{1}, \ldots, \sigma_{i} . r, \ldots, \sigma_{n}\right) \in \operatorname{Sem}(\mathbb{S})$. As $\operatorname{Sem}(\mathbb{S}) \subseteq \operatorname{OTraces}\left(\Sigma_{S y s}\right)$, we have that $\left(\sigma_{1}, \ldots \sigma_{i} . r, \ldots, \sigma_{n}\right) \in \operatorname{OTraces}\left(\Sigma_{S y s}\right)$. Assume that $i, r$ and $\left(\sigma_{1} \ldots, \sigma_{i}, \ldots \sigma_{n}\right)$ are chosen to be minimal and so $\sigma_{j} \in \operatorname{TTraces}\left(\mathbb{A}_{j}\right)$ for all $1 \leq j \leq n$. Thus, since $\left(\sigma_{1}, \ldots \sigma_{i}, \ldots, \sigma_{n}\right) \in$ $\operatorname{OTraces}\left(\Sigma_{S y s}\right)$, we have that $\left(\sigma_{1} \ldots, \sigma_{i}, \ldots \sigma_{n}\right) \in \operatorname{OTraces}($ Sys $)$. By Definition 8 , since $\mathbb{S}$ dtioco Sys we have that $\left(\sigma_{1} \ldots, \sigma_{i} . r, \ldots \sigma_{n}\right) \in \operatorname{OTraces}($ Sys $)$. Thus, $\left(\sigma_{1}, \ldots \sigma_{i} . r, \ldots, \sigma_{n}\right) \in \operatorname{TTraces}\left(\mathbb{A}_{1}\right) \times \ldots \times \operatorname{TTraces}\left(\mathbb{A}_{n}\right)$, and so we can deduce that $\sigma_{i} . r \in T$ Traces $\left(\mathbb{A}_{i}\right)$. This contradicts our hypothesis.

Now consider the right-to-left implication. The first condition of Definition 8 (dtioco) holds immediately from the hypotheses. We assume that we have been given $\left(\sigma_{1} \ldots \sigma_{i}, \ldots, \sigma_{n}\right) \in \operatorname{OTraces}($ Sys $), r \in \Sigma_{i} \cup D$ and $\left(\sigma_{1} \ldots, \sigma_{i} . r, \ldots \sigma_{n}\right) \in$ $\operatorname{Sem}(\mathbb{S})$ and are required to prove that $\left(\sigma_{1} \ldots, \sigma_{i} . r, \ldots \sigma_{n}\right) \in \operatorname{OTraces}(\operatorname{Sys})$.

Since $\left(\sigma_{1} \ldots, \sigma_{i} . r, \ldots \sigma_{n}\right) \in \operatorname{Sem}(\mathbb{S})$ we have that $\sigma_{i} . r \in \operatorname{TTraces}\left(\left.\mathbb{L}_{\mathbb{S}_{i}}\right|_{\mathbb{S}}\right)$. Further, since $\left(\sigma_{1} \ldots \sigma_{i}, \ldots, \sigma_{n}\right) \in \operatorname{OTraces}(\operatorname{Sys})$ we know that $\sigma_{i} \in \operatorname{TTraces}\left(\mathbb{A}_{i}\right)$. Thus, since $\left.\mathbb{L S}_{i}\right|_{\mathbb{S}}$ tioco $\mathbb{A}_{i}$, we have that $\sigma_{i} . r \in \operatorname{TTraces}\left(\mathbb{A}_{i}\right)$. Since $\operatorname{Sem}(\mathbb{S}) \subseteq$ $\operatorname{OTraces}\left(\Sigma_{S y s}\right)$ we have that $\left(\sigma_{1} \ldots \sigma_{i} . r, \ldots, \sigma_{n}\right) \in \operatorname{OTraces}\left(\Sigma_{S y s}\right)$. We thus have that $\left(\sigma_{1} \ldots \sigma_{i} . r, \ldots, \sigma_{n}\right) \in \operatorname{OTraces}\left(\Sigma_{\text {Sys }}\right), \sigma_{i} . r \in \operatorname{TTraces}\left(\mathbb{A}_{i}\right)$ and $\sigma_{j} \in$ $\operatorname{TTraces}\left(\mathbb{A}_{j}\right)$ for all $1 \leq j \leq n$ with $j \neq i$. From the definition of $\operatorname{OTraces}(\operatorname{Sys})$, we conclude that $\left(\sigma_{1} \ldots, \sigma_{i} . r, \ldots \sigma_{n}\right) \in \operatorname{OTraces}(S y s)$ as required.

\subsection{TIOSTSs as Symbolic Denotation of TIOSTs}

Now, we briefly present symbolic versions of TIOTSs that will be used to illustrate our test framework with a reasonable example. Indeed, generally, for expressiveness sake, we do not directly use TIOTSs, which are appropriate to theoretically reason about conformance, but do not permit real time constraints to be expressed. Here, we use TIOSTS (Timed Input Output Symbolic Transition Systems) [14, 15] which are automata that have variables to abstractly denote system states (we call them data variables) and variables to capture timing constraints (we call them clocks) on system executions.

TIOSTS introduce transitions of the form $\left(q, \mathcal{T}, \phi, \psi, a c t, \rho, q^{\prime}\right)$ where (i) $q$ and $q^{\prime}$ are states, $\phi$ and $\psi$ are guards on time and data respectively, (ii) $\mathcal{T}$ is a set of 
clocks to be reset to 0 when the action act occurs, (iii) act may be receptions of the form $c ? x(c ?)$ where $c$ is a channel and $x$ is a data variable or emissions of the form $c ! t(c !)$ where $t$ is a term and (iv) $\rho$ is an assignment of data variables denoting updates of variable values. Such a transition can be executed from $q$ if $\psi$ holds and at any moment for which values of the clocks are such that $\phi$ holds (as for timed automata). At this moment, the action occurs (signals $c$ ? or $c$ ! or a reception of a value on $x$ if the action is $c ? x$ or emission of the current value of $t$ if the action is $c ! t)$. Clocks of $\mathcal{T}$ are reset and data variables are updated according to $\rho$. Finally, by successively executing all consecutive transitions, one may form all timed traces of a TIOST.

\subsection{Example}

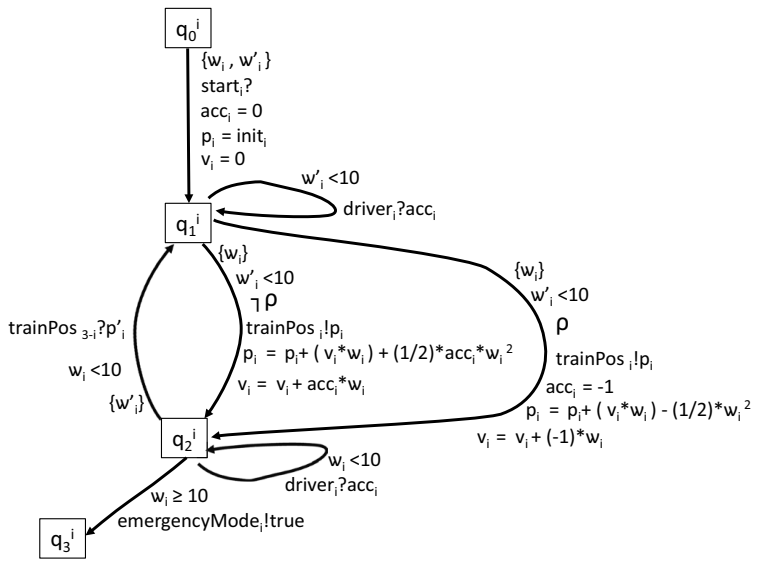

With: init $_{1}=42$ and init ${ }_{2}=300$ and $\rho \equiv p_{i}<p_{i}^{\prime} \leq\left(v_{i} * 20\right)+200$

Fig. 1. Train Control System Example: $T L C_{i}$ for $i=1,2$

Figure1 gives a simplified model of the functional requirements of Train Local Controllers (TLCs) that forms part of the European Train Control System 7 . Similar specifications can found, e.g. in 20] and in 21. The overall Train Control System (TCS) contains two Train Local Controllers (TLCs), one per train (say train 1 and train 2), going in the same direction on a rolling stock.

The symbol $i$ in Figure 1 should be replaced by two possible values, 1 and 2. The system $T C S=\left(T L C_{1}, T L C_{2}\right)$ ensures that the train on the rear side automatically decreases speed as soon as the one in front of it is too close. The relative position of trains is given by their positions, which can be accessed by consulting the value of variable $p_{i}$ : if $p_{1}<p_{2}$, then train 1 is behind train 2 .

\footnotetext{
${ }^{7}$ Interested readers can consult http://www.uic.org/spip.php?rubrique850
} 
The $T L C_{i}$ are automata containing 4 states $\left(q_{0}^{i}\right.$ the initial state, $q_{1}^{i}, q_{2}^{i}$ and $\left.q_{3}^{i}\right)$, communicating through channels $\left(\right.$ start $_{i}$, driver $_{i}$, emergency Mode $_{i}$ for communicating with the environment, $\operatorname{trainPos}_{i}$ for sending internal messages and $\operatorname{trainPos}_{3-i}$ for receiving internal messages) and having 4 data variables $\left(a c c_{i}\right.$ in $\{-1,0,1\}$ for the acceleration of the train, $v_{i}$ for the speed of the train, $p_{i}$ for the position value of the train, $p_{i}^{\prime}$ for the estimation of the position of the other train) and 2 clocks $\left(w_{i}\right.$, which is reset at each emission of the position and $w_{i}^{\prime}$, which is reset at each reception of the position of the second train).

$T L C_{i}$ specifies the following behaviour: after an initialisation phase, the train of interest sends its position to the other train, and in return, the other train is supposed to send its position. In this loop, two consecutive communication actions are supposed to be separated by a delay of less than 10 units of time. If the remote train does not send its position on time, the local train goes into an emergency mode (not detailed here). At any moment in the loop, the driver may ask to modify the train acceleration. The new value is taken into account only if it does not affect the safety of the system (safety is threatened if condition $\rho$ holds, that is, the distance between trains is less than the distance that can be covered by the rear train with the current acceleration). If safety is threatened, than the acceleration of the rear train is set to -1 in order to reduce its speed. Here are some examples of couples $\left(\sigma_{1}, \sigma_{2}\right)$ with $\sigma_{i}$ a trace of $T L C_{i}$ for $i$ in 1,2 :

- $\left(\sigma_{1}^{1}=\right.$ start $_{1}$ ?.(2).(1).driver ? $_{1}$ ?1.(1).(2).trainPos 1 142.(2).(3).trainPos 2 ?300.(2).(1), $\sigma_{2}^{1}=$ start $_{2}$ ?.(1).(1).trainPos 2 !300.(2).(2).trainPos 1 ?42.(3).(2).trainPos 2 !300.(1)) is a multitrace with the convention that numbers between parentheses are durations, numbers without parentheses are messages and the punctuation symbol "." separates durations and actions. Note that train 1 is the rear train and train 2 does not move. This multitrace corresponds to a situation in which all local testers have observed until the end of the behaviour of $T L C_{i}$ : in particular, all receptions have been preceded by an emission, and except the first duration (2) occurring in $\sigma_{1}^{1}$, all following durations (1).(1).(2).(2).(3).(2).(1) are the same in both traces (time elapsing property).

- $\left(\sigma_{1}^{2}=\right.$ start $_{1}$ ?.(2).(1).driver ${ }_{1} ? 1$, $\sigma_{2}^{2}=$ start $_{2}$ ?.(1).(1).trainPos 2 !300.(2).(2).trainPos 1 ?42.(3).(2).trainPos 2 !300.(1)) is such that $\sigma_{1}^{2}$ is a prefix of $\sigma_{1}^{1}$ and $\sigma_{2}^{2}=\sigma_{2}^{1} \cdot\left(\sigma_{1}^{2}, \sigma_{2}^{2}\right)$ is thus an observable multitrace. The observation of $T L C_{1}$ is stopped just after the driver asked to accelerate. Since the tester at $T L C_{2}$ observes for longer, $T L C_{2}$ receives the value 42 . So, $T L C_{1}$ sent its position (42), but the observer associated with $T L C_{1}$ did not wait long enough to record this action.

- $\left(\sigma_{1}^{3}=\right.$ start $_{1}$ ?.(2).(1).driver ${ }_{1}$ ?1.(1).(2).trainPos 1 !42.(1).trainPos 2 ?300.(1). trainPos 1 !60.(1).TrainPos 2 ?300,

$\sigma_{2}^{3}=$ start $_{2}$ ?.(1).(1).trainPos 2 !300.(2).(2).trainPos Pra2.(3).(2).trainPos $_{2}$ !300.(1)) does not constitute an observable multitrace. Indeed, in $\sigma_{1}^{3}$, $\operatorname{trainPos}_{1} ! 42$ is emitted and 3 units of time later, $\operatorname{trainPos} 2$ ?300 is received a second time. This reception corresponds to the second emission $\operatorname{trainPos}_{2}$ !300 in $\sigma_{2}^{3}$. Now, in $\sigma_{2}^{3}$, the second emission trainPos $2 ! 300$ occurs 5 units of time after 
the reception trainPos ? $_{1}$. This contradicts the time elapsing property: indeed, for $T L C_{1}$, time elapses of only 3 units of time between emission of 42 and reception of 300 , but, meanwhile, for $T L C_{2}$, it elapses for at least 5 units of time since it comprises the duration separating the reception of 42 and the emission of 300 .

\section{Implementing Distributed Timed Testing}

This section describes how testing for dtioco can be carried out in a manner that reflects our underlying assumptions and also utilises our compositionality result.

\subsection{Architecture}

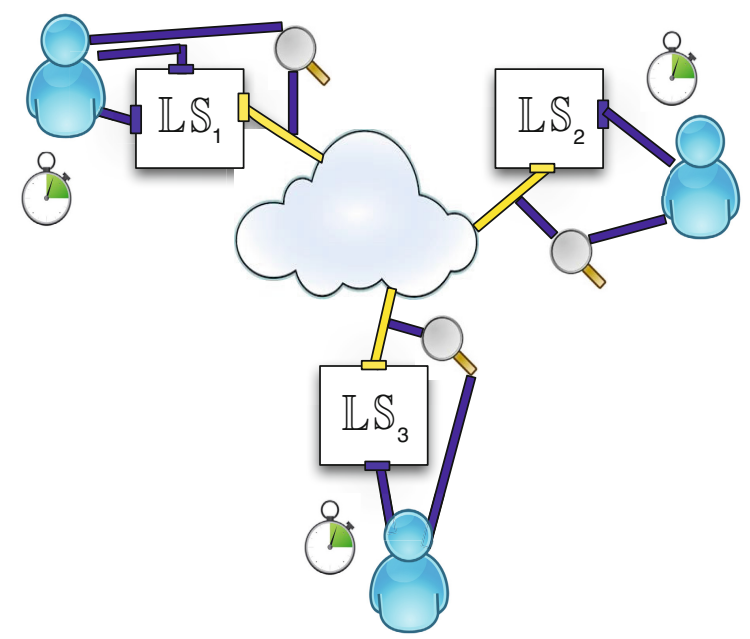

Fig. 2. Distributed testing Architecture

Figure 2 illustrates the architecture used. The SUT $\mathbb{S}$ is composed of LUTs $\mathbb{L S}_{1}, \mathbb{L S}_{2}$ and $\mathbb{L}_{3}$. Each LUT $\mathbb{L S}_{i}$ has channels connected to the environment (dark connections) and internal channels to exchange values with other LUTs $\mathbb{L S}_{i}$ (light connections). A tester $\mathbb{T}_{i}$ is associated with each LUT $\mathbb{L} \mathbb{S}_{i}$ and $\mathbb{T}_{i}$ may control inputs and observe outputs occurring on channels connected to the environment. The tester may also observe values sent through internal channels (represented by the magnifying glasses). Each $\mathbb{L} \mathbb{S}_{i}$ executes in a centralised way, so that the local tester can observe the order of actions occurring on its channels and can measure durations between consecutive actions. Therefore, behaviours observed by each $\mathbb{T}_{i}$ can be viewed as timed traces and may be analysed with respect to the set of timed traces of the model specifying the LUT. We cannot directly combine the timed traces observed at different LUTs since there is no global clock. Internal communications, represented by a cloud, are observed twice, at the emission and at the reception, by different testers. Recall, however, that we assume that all testers use clocks progressing at the same rate. 


\subsection{Process}

Consider SUT $\mathbb{S}=\left(\mathbb{L S}_{1}, \ldots, \mathbb{L}_{n}\right)$ with tester $\mathbb{T}_{i}$ at each $\mathbb{L}_{i}$ ( $i$ in $\left.1 . . n\right)$, that we want to test against system $S y s=\left(\mathbb{A}_{1}, \ldots, \mathbb{A}_{n}\right)$. Based on Definition 8 . and Property 1, we see that any fault of the whole system can be identified either at the level of one LUT, or at the level of internal communications: $(\mathbb{S}$ dtioco Sys $) \Longleftrightarrow\left(\left(\forall i \leq n,\left.\mathbb{L}_{\mathbb{S}_{i}}\right|_{\mathbb{S}}\right.\right.$ tioco $\left.\left.\mathbb{A}_{i}\right) \wedge \operatorname{Sem}(\mathbb{S}) \subseteq \operatorname{OTraces}\left(\Sigma_{\text {Sys }}\right)\right)$

So, if a local tester $\mathbb{T}_{i}$ exhibits timed trace $\sigma_{i}$ contradicting $\left.\mathbb{L}_{i}\right|_{\mathbb{S}}$ tioco $\mathbb{A}_{i}$, or if $\left(\sigma_{1}, \ldots, \sigma_{n}\right)\left(\sigma_{i}\right.$ observed by tester $\left.\mathbb{T}_{i}\right)$ does not form an observational trace, then ( $\mathbb{S}$ dtioco Sys) does not hold. Moreover, as these conditions are sufficient to ensure that $\mathbb{S}$ dtioco Sys, this suggests a two step testing process:

(1) Timed unitary testing of each $\mathbb{L} \mathbb{S}_{i}$ w.r.t. $\mathbb{A}_{i}$. Tester $\mathbb{T}_{i}$ associated with $\mathbb{L} \mathbb{S}_{i}$ checks that the timed trace it observes is allowed by $\mathbb{A}_{i}$ under tioco. If one of the testers reveals an error, then $(\mathbb{S}$ dtioco Sys) does not hold;

(2) Testing of internal communications. All the $\mathbb{T}_{i}$ keep track of the localised timed traces $\sigma_{i}$ observed. Tuple $\left(\sigma_{1}, \ldots, \sigma_{n}\right)$ of timed traces is analysed to check whether or not it constitutes an observational trace. If not, then ( $\mathbb{S}$ dtioco Sys) does not hold.

For the first step, the test execution process corresponds to classical unitary testing since $\mathbb{T}_{i}$ interacts with $\mathbb{L S}_{i}$ in the context of the whole system (modeled as $\left.\left.\mathbb{L} \mathbb{S}_{i}\right|_{\mathbb{S}}\right)$. The only slight difference is that inputs on internal channels are not controlled by the tester but by the remaining part of the system (the $\mathbb{L S}_{j}$ with $j \neq i$ ) and the cloud network. This kind of test architecture has already been addressed, typically in the context of orchestrations, a particular class of web services compositions. An orchestrator can be seen as a main localised module that orchestrates exchanges between a user and some web services. In [22, we proposed an (untimed) algorithm to test an orchestrator in the context of the system in which they are plugged. In such a test architecture, the orchestrator receives inputs from a user and web services: typically, the latter are not controlled by the tester. An orchestrator is an LUT and a whole orchestration can be seen as a special case of systems addressed in this paper. The algorithm in [22] can be adapted to be used in Step (1) to incorporate timed aspects, for example by considering the on-line test generation algorithm given in [14], or an off-line alternative [15] and by adapting them in order to ignore any duration that occurs before the first action of a timed trace. In fact, in our test framework, the off-line approach should be preferred, since for the moment, we have not addressed the question of test case generation, but focus mainly on the oracle problem. So, as we only need an algorithm for analysing trace conformance, it can be obtained for free from an off-line testing architecture.

Due to the lack of space, we do not fully present Step (1), and rather focus on the analysis of the system multitraces to check the observational trace property, and thus to achieve Step (2). 


\subsection{Observational Multitrace Checking}

The algorithm (Figure 3) checking the observable multitrace property follows the points of Definitions 3 and 4. In the sequel we assume that any duration observed in any timed trace consists of sequences of 1 unit (which implies that we can see all delays between communication actions as integers). This is exactly what is done in practice in a timed testing process, when using a clock to measure time between actions in a testing process (the clock itself imposes the basic delay defining the unity). The main idea is to store a multitrace $\left(\sigma_{1}^{c}, \ldots, \sigma_{n}^{c}\right)$ that is observed at the end of a run, to read it from the beginning and to store the elements already read in a multitrace ot $=\left(\mu_{1}, \ldots, \mu_{n}\right)$ while keeping the elements still to be read in a multitrace $m t=\left(\sigma_{1}, \ldots, \sigma_{n}\right)$. The algorithm check $(m t, o t)$ ends with success (return True) when the current multitrace $m t=\left(\sigma_{1}, \ldots, \sigma_{n}\right)$ to be analysed is the empty multitrace $(\varepsilon, \ldots, \varepsilon)$ (line 5$)$ and the read multitrace ot $=\left(\mu_{1}, \ldots, \mu_{n}\right)$ corresponds to the complete initial multitrace $\left(\sigma_{1}^{c}, \ldots, \sigma_{n}^{c}\right)$. There are two ways of reading a multitrace $\left(\sigma_{1}, \ldots, \sigma_{n}\right)$ : either there exists a trace $\sigma_{i}$ beginning with an action $a_{i}$ (Case (1)) (line 8), or a duration $d$ can be read on all admissible traces (Case (2)) (line 13):

Case (1). An action $a_{i}$ can be read by the algorithm from $\sigma_{i}$ (line 12), that is, removed from the trace still to be read (replace $\left(m t, i, \operatorname{tail}\left(\sigma_{i}\right)\right)$ and added to the trace already read (replace $\left(\right.$ ot $\left., i, \operatorname{addEnd}\left(\mu_{i}, a_{i}\right)\right)$ ) if one of the following conditions is fulfilled: (1) $a_{i}$ is an emission towards the environment or other subsystems (isOuptut $\left.\left(a_{i}\right)\right),(2) a_{i}$ is a reception from the environment (isInputEnv $\left.\left(a_{i}\right)\right)$, (3) $a_{i}$ is a reception of a message $m$ on the channel $c$ coming from one of the other subsystems $\left(\operatorname{isInputInt}\left(a_{i}\right)\right)$ and the number of occurrences of $a_{i}$ in $\mu_{i}$ (elements already read by the algorithm for the subsystem $\mathbb{L} \mathbb{S}_{i}$ ) is strictly less than the number of emissions already read by the algorithm, i.e. the number of $c ! m$ occurring in $\left(\mu_{1}, \ldots, \mu_{n}\right)\left(N b_{-} I\left(\mu_{i}, a_{i}\right)<N b_{-} O\left(o t, a_{i}\right)\right)$, provided that none of the subsystems $\mathbb{L S}_{j}(i \neq j)$ that can emit on the channel $c$ has a trace fully read, i.e. $\forall j \mid \mathbb{L S}_{j}$ can emit on c, $\sigma_{j} \neq \varepsilon$, and lastly, (4) $a_{i}$ is a reception of a message $m$ on the channel $c$ coming from one of the other subsystems and there exists a subsystem $\mathbb{L} \mathbb{S}_{j}(i \neq j)$ that can emit on the channel $c$ whose trace is already fully read, i.e. $\exists j \mid \mathbb{L} \mathbb{S}_{j}$ can emit on c , $\sigma_{j}=\varepsilon$.

In the algorithm, the predicate FullR(Chan $\left.\left(a_{i}\right), m t\right)$ is True when for all $j$ such that $\mathbb{L S}_{j}$ can emit on $\operatorname{Chan}\left(a_{i}\right), \sigma_{j}$ (occurring in $\left.m t=\left(\sigma_{1}, \ldots, \sigma_{n}\right)\right)$ is equal to $\varepsilon$. The predicate is used both for subcases (3) and (4).

Case (2). A duration $d=1$ can be read by the algorithm if one of the non empty traces $\sigma_{i}$ starts with a duration $d_{i}>0$ and if for all traces $\sigma_{i}$ starting with an action, the reading of the trace has not been started, i.e. $\mu_{i}=\varepsilon$ (TimeElapsing $(m t, o t)$ ). In this case, the duration $d$ is subtracted from all durations $d_{i}$ occurring at the beginning of traces $\sigma_{i}\left(d_{i}\right.$ is simply removed if $\left.d_{i}=1\right)$ and added to the corresponding $\mu_{i}($ time_elapse_of_1 $1(m t, o t))$. 
If the reading cannot be continued until reaching the empty multitrace, then the initial multitrace does not meet the targeted observable multitrace property (check $(m t, o t)$ returns the value False, initialised at line 7). As the underlying principle consists of considering all possible configurations for interleaving emissions and receptions of different subsystems, its complexity is clearly high. However, as the algorithm is applied only once the local traces are completely stored (off-line algorithm), a good efficiency is not of primary necessity.

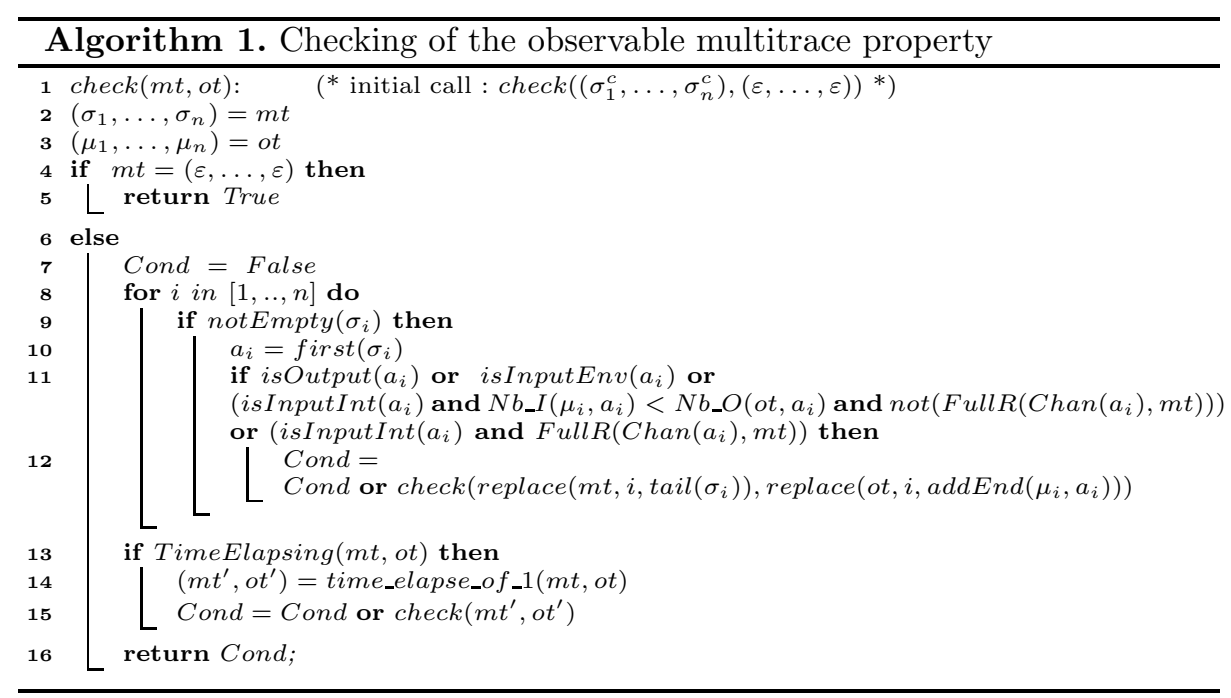

Fig. 3. Checking Observable Trace property

\section{Conclusions}

There has been growing interest in distributed testing where the SUT has physically distributed interfaces, there is a separate tester at each interface and a tester only observes the interactions at its interface. This paper extends previous work by investigating distributed testing from specifications based on TIOTSs that interact through an implicit distributed protocol. We assume that the SUT and specification are both composed of separate components at the interfaces and that the sending and receiving of messages between components is observed during testing. Components themselves are described as TIOTS. We define the semantics of such systems as a sets of tuples containing a local trace for each interface. Such tuples, called observational multitraces, have to respect consistency conditions ensuring that all local traces together reflect correct interactions between components. Having defined observations, we defined an implementation relation dtioco for distributed testing. This implementation relation is an extension of tioco (timed version of ioco). It captures two things: tuples resulting from 
distributed interactions with the system under test are valid (i.e. are observational multitraces), and all reactions of the system under test after a specified multitrace should also be specified.

We also provide a compositionality result, which shows that an SUT conforms to a specification under dtioco if and only if all of the observations of the SUT that can be made are valid and the local projections of the SUT conform to the corresponding components of the specification under tioco. This result allows us to reuse techniques developed for tioco. We then describe how testing can be implemented and give an algorithm that checks that an observation is valid.

Since this is the first work to define an implementation relation for distributed timed testing, there are several lines of future work. First, there is a need to define and implement suitable test generation algorithms. In particular, it is necessary to define distributed test purposes, and to find test generation strategies to drive system executions so that they follow those test purposes. Recent work has shown that for untimed systems it is undecidable whether there is a distributed test case guaranteed to force a model $M$ into a given state $s$ or to distinguish two states and that this holds even if we restrict $M$ to being a deterministic finite state machine (DFSM) [4]. Thus, general test generation problems are likely to be undecidable but we will investigate conditions under which they are decidable. Such test generation problems become tractable if we restrict attention to controllable test cases and DFSM models [8] and so it would be interesting to investigate notions of controllability for timed models. Second, we assume that local clocks progress at the same rate; it should be possible to generalise the results to the case where the clocks can drift but, for example, we have upper bounds on the rate of drift. We also intend to consider the case where the sending and receiving of internal messages are hidden.

\section{References}

1. ISO/IEC JTC 1, J.T.C.: International Standard ISO/IEC 9646-1. Information Technology - Open Systems Interconnection - Conformance testing methodology and framework - Part 1: General concepts. ISO/IEC (1994)

2. Dssouli, R., von Bochmann, G.: Error detection with multiple observers. In: Protocol Specification, Testing and Verification V, pp. 483-494. Elsevier Science, North Holland (1985)

3. Dssouli, R., von Bochmann, G.: Conformance testing with multiple observers. In: Protocol Specification, Testing and Verification VI, pp. 217-229. Elsevier Science, North Holland (1986)

4. Hierons, R.M.: Reaching and distinguishing states of distributed systems. SIAM Journal on Computing 39(8), 3480-3500 (2010)

5. Luo, G., Dssouli, R., von Bochmann, G.: Generating synchronizable test sequences based on finite state machine with distributed ports. In: The 6th IFIP Workshop on Protocol Test Systems, pp. 139-153. Elsevier, North-Holland (1993)

6. Sarikaya, B., von Bochmann, G.: Synchronization and specification issues in protocol testing. IEEE Transactions on Communications 32, 389-395 (1984)

7. Ural, H., Wang, Z.: Synchronizable test sequence generation using UIO sequences. Computer Communications 16(10), 653-661 (1993) 
8. Hierons, R.M., Ural, H.: The effect of the distributed test architecture on the power of testing. The Computer Journal 51(4), 497-510 (2008)

9. Brinksma, E., Heerink, L., Tretmans, J.: Factorized test generation for multiinput/output transition systems. In: FIP TC6 11th International Workshop on Testing Communicating Systems (IWTCS). IFIP Conference Proceedings, vol. 131, pp. 67-82. Kluwer (1998)

10. Hierons, R.M., Merayo, M.G., Núñez, M.: Implementation relations for the distributed test architecture. In: Suzuki, K., Higashino, T., Ulrich, A., Hasegawa, T. (eds.) TestCom/FATES 2008. LNCS, vol. 5047, pp. 200-215. Springer, Heidelberg (2008)

11. Hierons, R.M., Merayo, M.G., Núñez, M.: Implementation relations and test generation for systems with distributed interfaces. Distributed Computing 25(1), 35-62 (2012)

12. Hierons, R.M., Merayo, M.G., Núñez, M.: Using time to add order to distributed testing. In: Giannakopoulou, D., Méry, D. (eds.) FM 2012. LNCS, vol. 7436, pp. 232-246. Springer, Heidelberg (2012)

13. Khoumsi, A.: A temporal approach for testing distributed systems. IEEE Transactions on Software Engineering 28(11), 1085-1103 (2002)

14. Escobedo, J.P., Gaston, C., Le Gall, P.: Timed Conformance Testing for Orchestrated Service Discovery. In: Arbab, F., Ölveczky, P.C. (eds.) FACS 2011. LNCS, vol. 7253, pp. 133-150. Springer, Heidelberg (2012)

15. Bannour, B., Escobedo, J., Gaston, C., Gall, P.L.: Off-line test case generation for timed symbolic model-based conformance testing. In: Nielsen, B., Weise, C. (eds.) ICTSS 2012. LNCS, vol. 7641, pp. 133-150. Springer, Heidelberg (2012)

16. Gaudel, M.C.: Testing can be formal too. In: Mosses, P.D., Nielsen, M. (eds.) CAAP 1995, FASE 1995, and TAPSOFT 1995. LNCS, vol. 915, pp. 82-96. Springer, Heidelberg (1995)

17. Krichen, M., Tripakis, S.: Black-box time systems. In: Proc. of Int. SPIN Workshop Model Checking of Software. Springer (2004)

18. Bohnenkamp, H.C., Belinfante, A.: Timed Testing with TorX. In: Fitzgerald, J.S., Hayes, I.J., Tarlecki, A. (eds.) FM 2005. LNCS, vol. 3582, pp. 173-188. Springer, Heidelberg (2005)

19. Schmaltz, J., Tretmans, J.: On Conformance Testing for Timed Systems. In: Cassez, F., Jard, C. (eds.) FORMATS 2008. LNCS, vol. 5215, pp. 250-264. Springer, Heidelberg (2008)

20. Meyer, R., Faber, J., Hoenicke, J., Rybalchenko, A.: Model checking duration calculus: a practical approach. Formal Asp. Comput. 20(4-5), 481-505 (2008)

21. Andres, C., Yevtushenko, N., On, A.R.C.: modeling and testing the european train control system. Technical Report TechRca 14-03-2013, Telecom Sudparis (2013)

22. Escobedo, J., Gaston, C., Gall, P.L., Cavalli, A.R.: Testing web service orchestrators in context: A symbolic approach. In: Int. Conf. SEFM. IEEE (2010) 